\title{
The role of echocardiography in the management of adult patients with congenital heart disease following operative treatment
}

\author{
Kálmán Havasi, Nóra Ambrus, Anita Kalapos, Tamás Forster, Attila Nemes \\ 2nd Department of Medicine and Cardiology Centre, Medical Faculty, Albert Szent-Györgyi Clinical Centre, University of Szeged, Szeged, \\ Hungary \\ Contributions: (I) Conception and design: K Havasi; (II) Administrative support: N Ambrus, A Kalapos; (III) Provision of study materials or patients: \\ All authors; (IV) Collection and assembly of data: K Havasi; (V) Data analysis and interpretation: K Havasi, N Ambrus, A Kalapos; (VI) Manuscript \\ writing: All authors; (VII) Final approval of manuscript: All authors. \\ Correspondence to: Attila Nemes, MD, PhD, DSc, FESC. 2nd Department of Medicine and Cardiology Centre, Medical Faculty, Albert Szent-Györgyi \\ Clinical Centre, University of Szeged, H-6725 Szeged, Semmelweis street 8, Szeged, Hungary. Email: nemes.attila@med.u-szeged.hu.
}

\begin{abstract}
Treatment of congenital heart diseases has significantly advanced over the last few decades. Due to the continuously increasing survival rate, there are more and more adult patients with congenital heart diseases and these patients present at the adult cardiologist from the paediatric cardiology care. The aim of the present review is to demonstrate the role of echocardiography in some significant congenital heart diseases.
\end{abstract}

Keywords: Echocardiography; congenital heart disease; adult

Submitted Jul 03, 2018. Accepted for publication Sep 07, 2018.

doi: $10.21037 / \mathrm{cdt} .2018 .09 .11$

View this article at: http://dx.doi.org/10.21037/cdt.2018.09.11

\section{Introduction}

Treatment of congenital heart diseases has significantly advanced during the last few decades. Due to improving cardiac surgical techniques and perioperative care, many children - who would have been considered to be incurable before-survive to adulthood (1). Surgical techniques include palliative treatments performed so that the patient can survive, and curative methods performed to restore complete anatomical or hemodynamic status. Recently, curative surgeries performed at the earliest possible are considered to be the standard procedure (2). Improved survival rates result in more adult patients with congenital heart disease who present at the adult cardiologist from paediatric cardiology care. By the $21^{\text {st }}$ century, there are more adult patients with congenital heart diseases than children (3). The adult cardiologist should know the disease, its natural course, and the principle of the applied surgical method and the potential long-term complications of adult congenital heart disease (ACHD) patients for optimal therapy. In addition, adequate results may be provided only if the patient's condition is evaluated by the proper imaging modality. Therefore, the treating physician should know the benefits, disadvantages and clinical indications of the certain imaging modality. Selecting the proper imaging modality is essential in determining the severity of the clinical problem and the necessary interventions (2). In current clinical practice, the most often used non-invasive imaging method in this case is echocardiography. Ultrasound methods and some common congenital heart diseases will be reviewed below. As most disorders described in this review affect the right heart, the echocardiography section of this article will mostly consist of information regarding the echocardiography of the right heart.

\section{Echocardiographic techniques and modalities}

Echocardiography is a non-invasive imaging method that can be performed bedside, no radiation exposure occurs, it is an inexpensive method, and it can be repeated at any time. Echocardiography examines the heart from several windows [transthoracic echocardiography (TTE), 
transoesophageal echocardiography (TEE), intracardiac echocardiography (ICE)] and with several methods [M mode, two dimensional (2D), colour Doppler, tissue Doppler, real time three-dimensional (3D) and 3D speckle tracking] (4). In the routine practice, several transthoracic echocardiography methods are used for the follow-up of patients with congenital heart diseases. Two-dimensional echocardiography is used to measure the size and determine the location and function of the heart chambers, and to describe the origin and course of the large vessels. Colour Doppler echocardiography is indicated to describe flow parameters, stenoses, regurgitations, and to determine the severity of the flow abnormality, for example, it can be used to detect residual stenoses or insufficiencies. Continuous wave Doppler ultrasound can quantify the stenosis $(2,4)$. Examination of the right heart is essential in heart diseases accompanied by pressure or volume overload of the right heart as the consequence of the primary disease or a residual lesion (2). Right atrial area, transverse and longitudinal diameters of the right ventricle, right ventricular function, diameter of the inferior caval vein and changes in the diameter of the inferior caval vein with breathing have to be determined.

TEE is another window to the heart, a semi-invasive method, nowadays all the above mentioned methods are available in case of TEE as well. The chambers of the heart, the septa, the valves and the large vessels are examined directly from the oesophagus using a transducer inserted into the oesophagus. There are several valvular diseases in the diagnosis of which the sensitivity and specificity of transoesophageal echocardiography is much better compared with transthoracic echocardiography, including bicuspid aortic valve, detection of vegetations and residual septal defects and guiding interventions of these defects (2,5-7).

From the novel methods, real-time 3D echocardiography is suitable to visualize anatomical structures while moving in space. For example, if an ASD is detected with 2D colour Doppler echocardiography, 3D echocardiography is able to show the shape of the defect and its location compared with other anatomical structures, which is helpful in planning potential interventions or surgeries (7). Spatial visualization of anatomical structures allows proper evaluation of flow parameters. Nowadays, 3D echocardiography is routinely used to evaluate septal defects and coarctation of the aorta (CoA) $(2,7,8)$. Transoesophageal 3D echocardiography is especially helpful in describing valve diseases and intraoperatively, it allows selection of the optimal valve plasty and evaluation of the efficacy of the surgery (9). 2D and 3D speckle tracking (strain) echocardiography is used for the objective and quantitative evaluation of the function of the heart chambers. Speckle-tracking echocardiography can detect impairment of pump function in congenital heart disease patients early (10-12).

Although it is not part of the routine clinical practice, and it is available only in specialized laboratories, intracardiac echocardiography is useful in the check-up of arrhythmia developing as a consequence of valve diseases and to explore the anatomy of the heart that has been operated on and modified even for several times. Intracardiac echocardiography may help determining the modified anatomy accompanying a certain arrhythmia substrate, enabling a potentially successful radiofrequency intervention (13). Echocardiography of some common congenital heart diseases will be discussed below. As the majority of the above described valve diseases lead to the overload of the right heart in the long-term, examination of the right heart will be described thoroughly in the methods.

\section{Most important congenital heart diseases}

\section{Atrial septal defect (ASD)}

ASD is one of the most common congenital heart diseases characterized by a left to right shunt in the level of the atria leading to volume overload in the right atrium (14). The atrial septum consists of two segments, the primum and secundum septum. None of these segments separate completely the two atria, by partially overlapping each other. Depending on the location of the lesion in the atrial septum, the ASD may be a primum or secundum type defect. In the long-term, large ASDs may lead to pulmonary hypertension and the left to right shunt may change and right to left shunt may develop (2). Echocardiography is a cardinal imaging method in determining the time and type of the surgery or intervention. Previously, ASDs were treated with surgery, recently, transcatheter closure of ASDs is available (15), for this intervention, thoracotomy or cardiac pump is not required. This poses a much smaller stress to the patient, and hospitalization can be significantly shorter. Size and function of the right heart should be closely monitored during the follow-up of patients undergoing transcatheter closure of an ASD to detect right heart overload developing as a consequence of a potential residual defect and left to right shunt. The aim of postoperative follow-up of these patients is to detect residual shunts and 


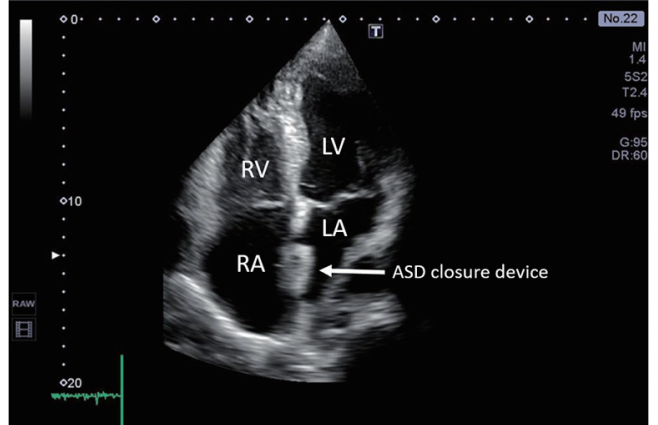

Figure 1 Atrial septal defect closure device: 2D echocardiography image from four-chamber view, the arrow shows the atrial septal defect closure device. LV, left ventricle; LA, left atrium; RV, right ventricle; RA, right atrium; ASD, atrial septal defect.

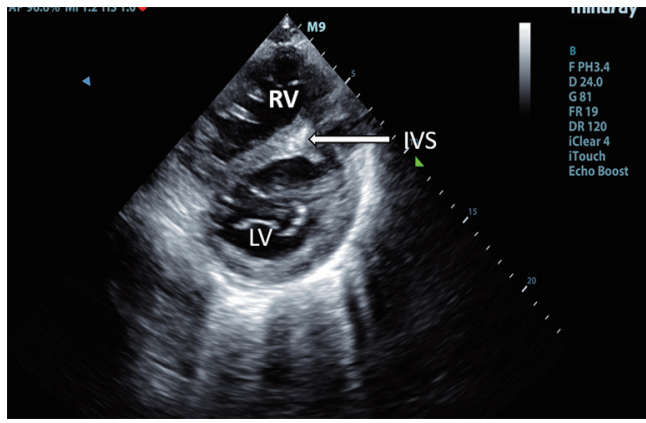

Figure 2 Diastolic D sign: the dilated right ventricle deviates the septum towards the left ventricle in parasternal short axis view, therefore cross section of the left ventricle is D-shaped (arrow shows the ventricular septum). LV, left ventricle; RV, right ventricle, IVS, interventricular septum.

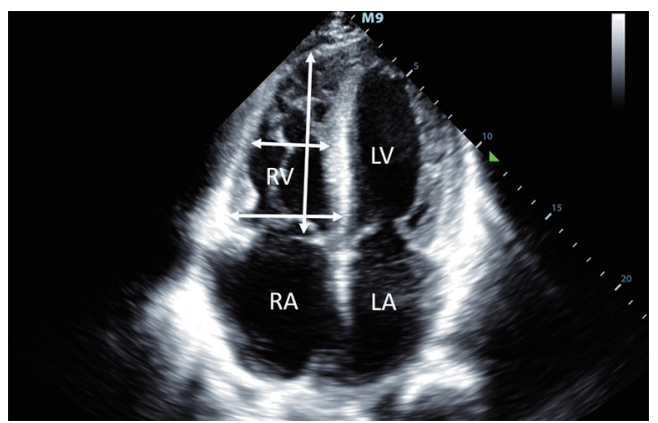

Figure 3 In order to measure the right ventricle, measurements are performed at the annulus, in the middle section of the ventricle in transverse direction and from the annulus to the apex of the right ventricle in longitudinal direction. Arrows show the diameters. LV, left ventricle; LA, left atrium; RV, right ventricle; RA, right atrium.

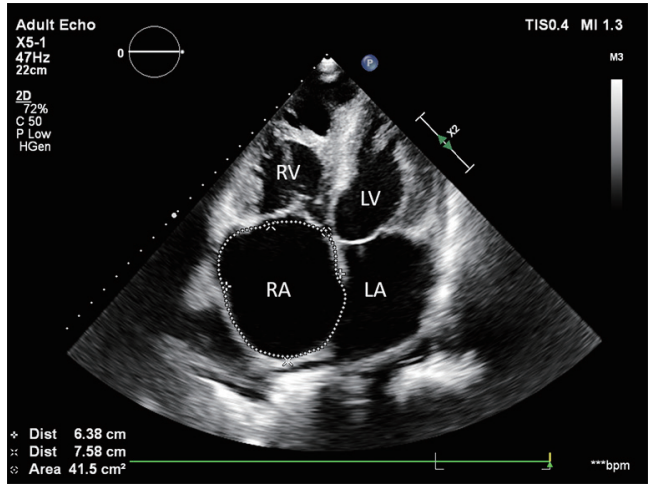

Figure 4 In order to determine the size of the right atrium, the right atrial area is calculated from the apical four-chamber view with planimetry (dashed line in the image). LV, left ventricle; LA, left atrium; RV, right ventricle; RA, right atrium.

to examine the status of the right heart (2). Transthoracic $2 \mathrm{D}$ echocardiography may be used to evaluate the location of the closure device (Figure 1), residual shunts are detected with colour Doppler method. Volume overload of the right ventricle may lead to a swift in the ventricular septum towards the left ventricle, as a consequence, the left ventricle will not be round-shaped, but will have a $\mathrm{D}$-shape in parasternal short axis view, this is the so-called diastolic D sign (Figure 2). 2D echocardiography may be used to measure the size of the right ventricle, right atrial area corrected to body surface area, TAPSE (tricuspid annular plane systolic excursion) (Figures 3-5), the diameter of the inferior vena cava and changes in the diameter with breathing, as well as pulmonary arterial pressure. Sensitivity of TEE is superior in detecting potential residual ASDs compared with TTE from subcostal view (16). The atrial septum should be examined from 0-45-60 degrees, and the bicaval view (90-110 degrees) may also be used.

\section{Ventricular septal defect (VSD)}

The ventricular septum has a muscular and membranous part. Depending on the location of the defect, there are membranous (subaortic), muscular and subpulmonary VSDs. Defects may be located in the inflow tract, near the tricuspid valve. Left to right shunt develops in the level of the ventricles. Potential complications include pulmonary hypertension and Eisenmenger's syndrome. In order to 
prevent these complications, large VSDs have to be closed in time (2). VSDs are most commonly closed by surgery with the implantation of a patch, or interventionally by an occluder, rarely small membranous defects are closed with direct sutures. During the follow-up of these patients, the position of the patch and potential residual defects can be visualized (Figure 6). Hemodynamic consequences of the disease, including the size of the right heart (right atrial area, transverse and longitudinal diameter of the right ventricle, diameter of the inferior vena cava and changes of the diameter with breathing, as well as flow in the hepatic veins) and the function of the right ventricle [TAPSE or FAC (fractionated area change)] have to be evaluated. Gradients measured in the residual VSD using

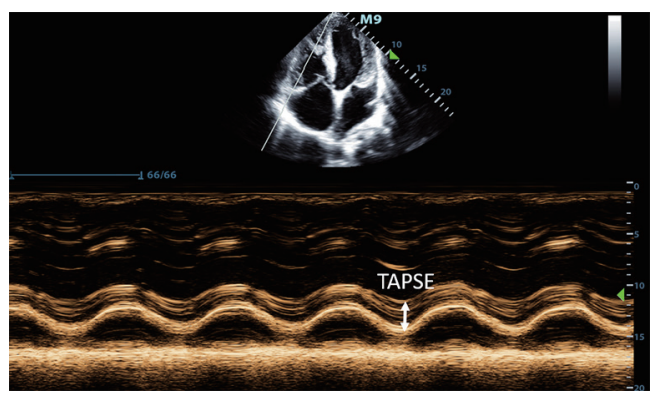

Figure 5 Tricuspid annular plane systolic excursion (TAPSE): in the apical four-chamber view, the excursion of the tricuspid annular plane is calculated measuring the distance of the tricuspid annular movement between end-diastole to end-systole in M-mode image (arrow in the image). continuous Doppler echocardiography well characterize the hemodynamic status and the extent of the shunt. A large maximum gradient above $100 \mathrm{mmHg}$ suggests a left to right shunt and normal right ventricular pressure. Decreasing gradient or change in the direction of the shunt (right to left shunt) confirms progression of the disease. Shunt volume may be determined with Doppler echocardiography, although image quality may be poor in certain patients, which significantly increases the risk of false results, one $\mathrm{mm}$ error in distance measurement leads to a multiplied error, as the following equitation is used to calculate shunt volume:

$\mathrm{Qp}=\mathrm{VTT}_{\mathrm{RVOT}} \times \pi \times\left(\mathrm{d}_{\mathrm{RVOT}} / 2\right)^{2}, \mathrm{Qs}=\mathrm{VTI}_{\mathrm{LVOT}} \times \pi \times\left(\mathrm{d}_{\mathrm{LVOT}} / 2\right)^{2}$

where the diameter (d) of the right ventricular outflow tract (RVOT), velocity time integral (VTI) of the flow via the RVOT, the diameter of the left ventricular outflow tract (LVOT) and the VTI of the LVOT are determined using 2D echocardiography and pulsed wave Doppler method respectively. Currently, cardiac MRI is the optimal method to measure $\mathrm{Qp} / \mathrm{Qs}$. If $\mathrm{Qp} / \mathrm{Q} s>1.5$, shunt volume is haemodynamically significant (17).

\section{Tetralogy of Fallot (TOF)}

Tetralogy of Fallot is the most common cyanotic congenital heart disease (18). It is characterized by VSD, overriding aorta, stenosis of the RVOT (that can be infundibular or valvular) and consequent right ventricular hypertrophy. The so called "blue baby syndrome" may clinically vary from slight to severe cyanosis. The first palliative surgery
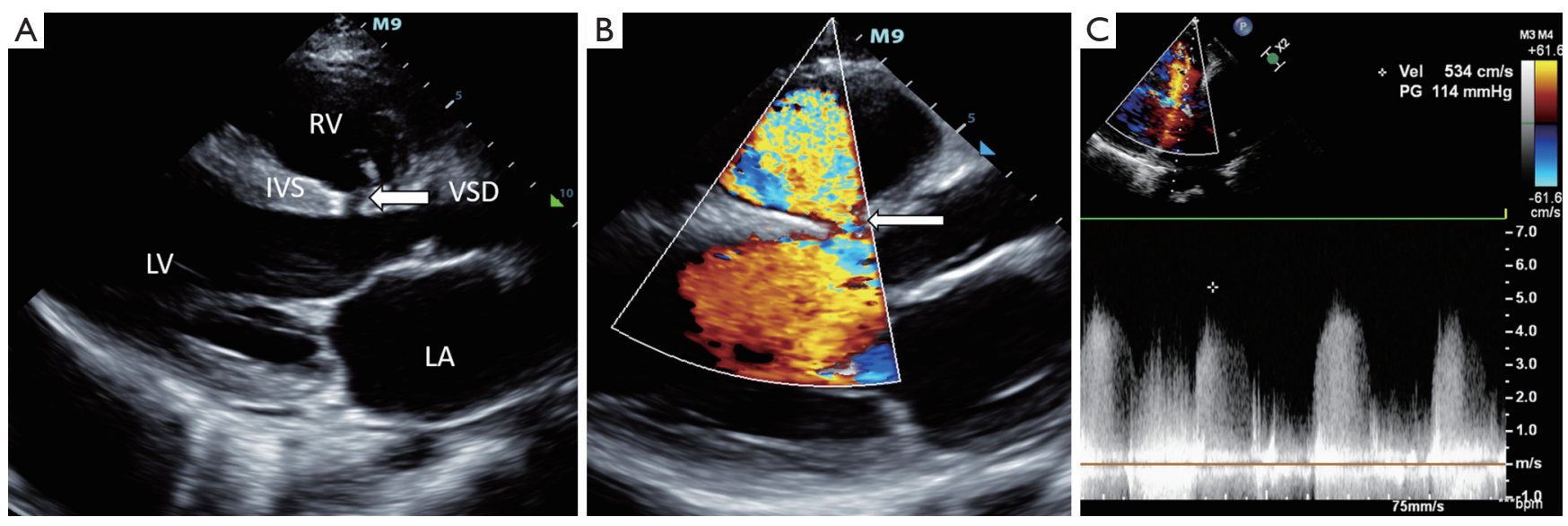

Figure 6 A membranous VSD is visible in the image. (A) Parasternal long axis view; (B) the same view with colour Doppler method; (C) pressure gradient between the two ventricles with continuous wave Doppler. LV, left ventricle; LA, left atrium; RV, right ventricle; IVS, interventricular septum; VSD, ventricular septal defect. 


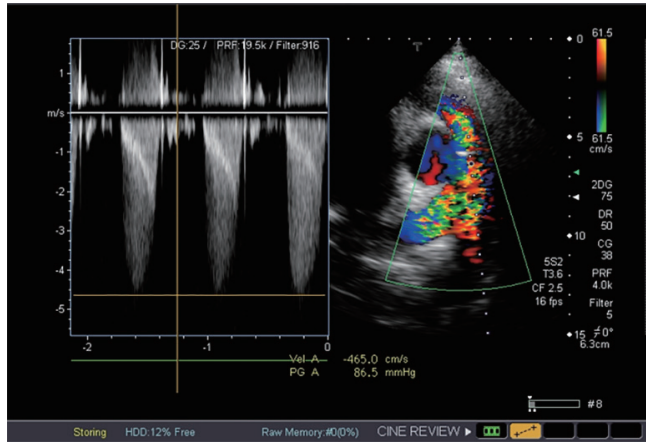

Figure 7 Parasternal short axis view of patient with tetralogy of Fallot who had cardiac surgery before shows turbulent flow in the right ventricular outflow tract with colour Doppler method. The gradient is determined with continuous wave Doppler echocardiography.

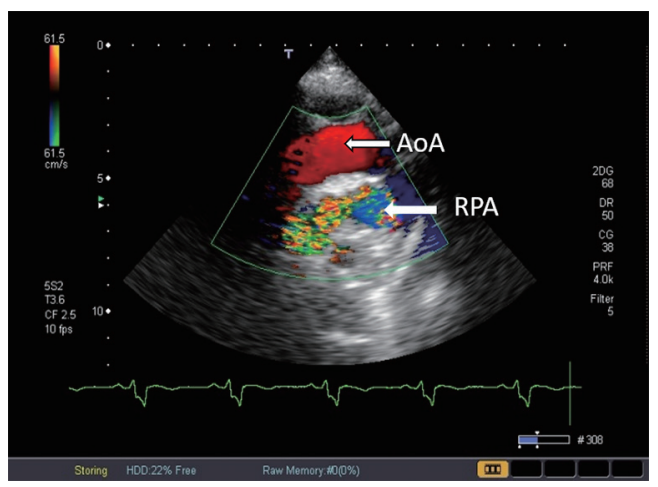

Figure 8 Suprasternal 2D image showing the aortic arch with laminar flow. The right pulmonary artery with turbulent flow is visualized below the aorta. AoA, aortic arch; RPA, right pulmonary artery.

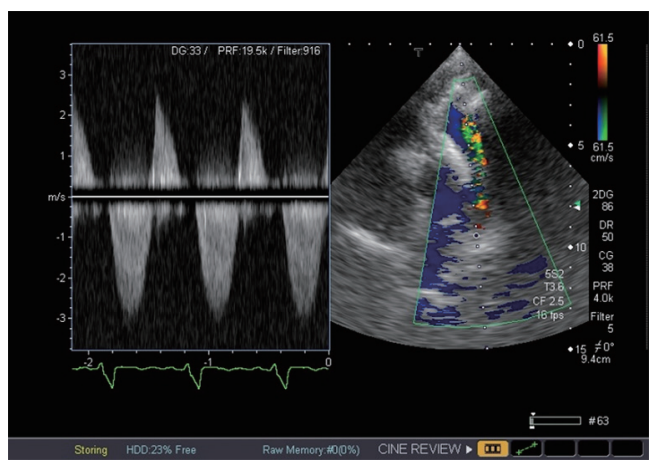

Figure 9 Pulmonary stenosis and insufficiency in surgically corrected tetralogy of Fallot. Continuous wave Doppler ultrasound is used in the right ventricular outflow tract. Doppler curve below the baseline shows restenosis, the curve above the baseline is indicating insufficiency. was performed on the 29 November 1944, the procedure was called Blalock Taussig-Thomas shunt. During this procedure, the hypoperfused pulmonary circulation is resolved with a systemo-pulmonary shunt providing perfusion in the pulmonary circulation. There are other systemo-pulmonary shunts, such as Pott's shunt or Waterston-Cooley shunt. Later, complete reconstruction surgery was performed during which the VSD is closed, the RVOT is reconstructed with the implantation of a homograft or a patch (18). Echocardiography is the recommended tool for the long-term follow-up of these patients in the clinical practice, the size and function of the right heart and potential progression of stenosis and insufficiency of the pulmonary valve are evaluated (Figures 7-9). During the check-up examination, the diameter and length of the right ventricle, TAPSE characterizing the function of the right ventricle FAC and right atrial area have to be determined using echocardiography in addition to evaluating the clinical parameters of the patients. Furthermore, diameter of the inferior vena cava and changes of the diameter with breathing, as well as flow in the hepatic veins measured from subcostal view provide important information (2). Dilated inferior vena cava above $20 \mathrm{~mm}$ in diameter, and a diameter not changing with breathing suggest increased pulmonary pressure. Follow-up of the right ventricular pressure calculated from the tricuspid insufficiency using continuous wave Doppler echocardiography is also important. Right heart dilatation and hypertrophy may develop as a consequence of residual pulmonary stenosis or insufficiency (2). Right atrial strain may be detected using 3DSTE (Figure 10). In the long-term, the treating cardiologist has to decide whether and when a reoperation is required, changes in the above parameters are very important factors in this decision. Therefore, these parameters have to be measured regularly, and have to be evaluated together with the functional status and complaints and symptoms. If progression is detected in the echocardiography parameters, cardiac MRI is recommended, the sensitivity and specificity of MRI is superior compared to echocardiography in evaluating the morphology and function of the right heart (2). Cardiac MRI can be used to determine right ventricular volumes in $\mathrm{ml}$ and pulmonary insufficiency can be quantified in \% (19).

\section{Dextro-transposition of the great arteries (TGA)}

(Dextro-)TGA is a very severe, complex congenital heart 
disease (20). In congenital TGA, the aorta arises from the right ventricle, while pulmonary artery from the left ventricle. Accordingly, there are two non-communicating circulations. This condition is compatible with life only if there is a connection between the two circulations, such as in the presence of an ASD, VSD, persistent ductus arteriosus or their combination (21). The primary treatment of TGA is to create or keep communication between the two circulations in early infancy (22). The socalled Rashkind's septostomy may be used to connect the two circulations, during this method, the atrial septum is fenestrated by a catheter balloon (23) immediately after

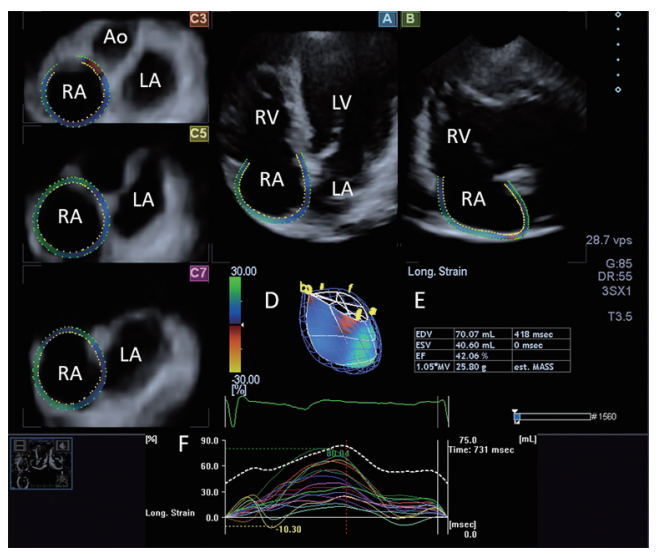

Figure 10 3DSTE right atrial modelling and measurements from apical four-chamber (A) and two-chamber (B), as well as from cross sectional (C: C3-5-7) views. (D) 3D model of the right atrium, (E) measured right atrial volumetric data, $(\mathrm{F})$ measured right atrial longitudinal strain curves of each segment. birth. Reconstruction surgeries performed since the late 1960s created a communication in the level of the atria (22). After these operations, the blood from the SVC and IVC is redirected to the left atrium and morphologically left (subpulmonary) ventricle, while blood from the pulmonary veins is channelled to the right atrium and morphologically right (systemic) ventricle. Notably, the right ventricle is pumping the blood to the aorta. The atrial switch surgeries had two surgical techniques, Senningand Mustard surgeries. If a large VSD and stenosis of the RVOT, the pulmonary valve and/or pulmonary artery were also associated with TGA, Rastelli-surgery is also an available option (24). During this procedure, the body of the right ventricle is connected to the transected pulmonary artery via a conduit, and the VSD is closed using a patch so that the aorta is originating from the left ventricle. As a result, the deoxygenated blood from the right ventricle gets to the pulmonary artery via the conduit, while the oxygenated blood is pumped from the aorta to the systemic circulation. Several questions have to be answered with echocardiography. In the atrial baffles, stenosis or leakage may occur, therefore follow-up echocardiography has to thoroughly evaluate flow in these structures (Figure 11). The "weaker" right ventricle has to provide the blood supply for the systemic circulation, therefore its pump function is an important data. TAPSE and FAC are those parameters that can be used to characterize the right ventricular function along with the $\mathrm{dP} / \mathrm{dt}$ value in case of tricuspid insufficiency. $\mathrm{dP} / \mathrm{dt}$ value characterizes the systolic function of the ventricle. From the 1990s, the above mentioned procedures have been replaced by the neonatal arterial switch surgery, where the two great arteries are transected

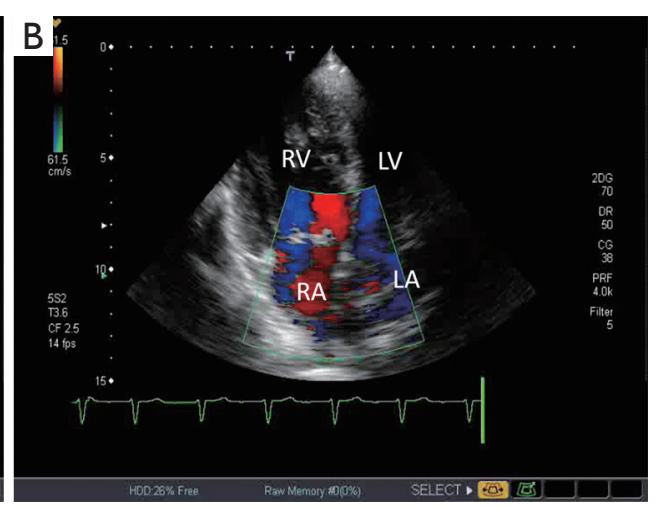

Figure 11 Status after Senning surgery. (A) Apical four-chamber view demonstrating a dilated, trabecularized right ventricle with a rudimentary left ventricle and the baffle; (B) apical four-chamber view with colour Doppler echocardiography. LV, left ventricle; LA, left atrium; RV, right ventricle; RA, right atrium. 

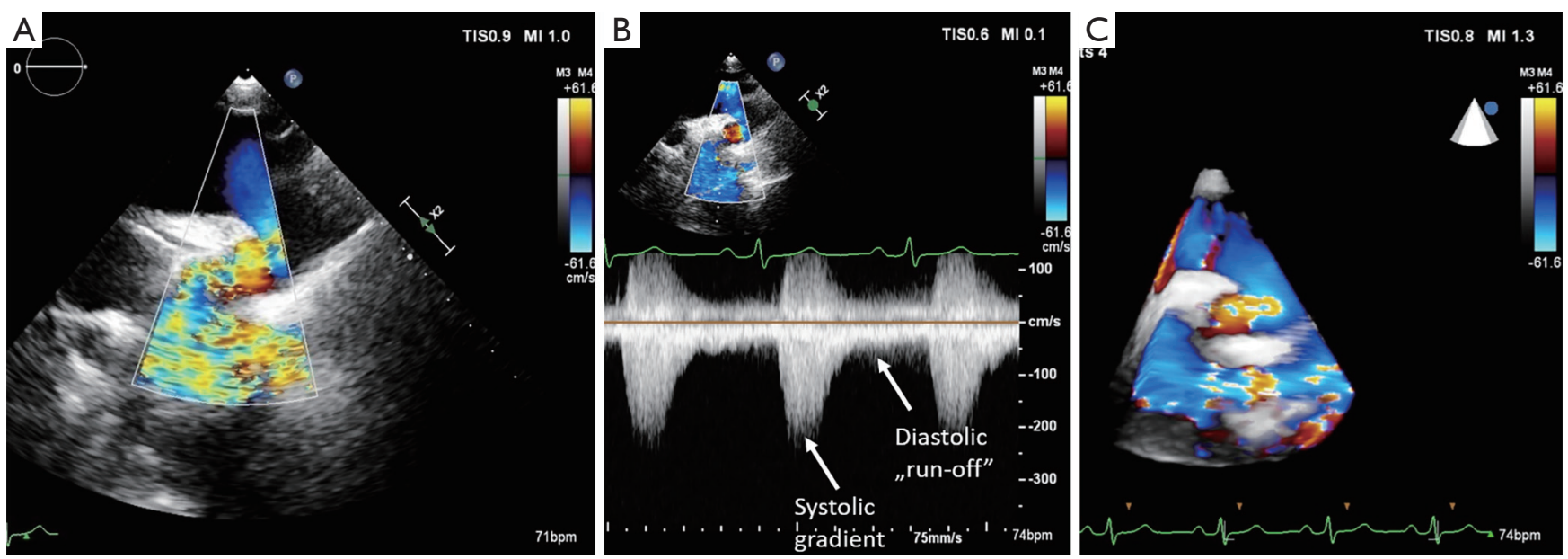

Figure 12 Restenosis of coarctation of the aorta after surgery. (A) Suprasternal view with 2D echocardiography and colour Doppler echocardiography demonstrating restenosis after surgery; (B) pressure gradient may be measured with continuous wave Doppler echocardiography and a slight run off phenomenon is visible; (C) real-time $3 \mathrm{D}$ echocardiography visualizing the spatial location and morphology of the restenosis.

and interchanged, then the coronary arteries are replaced to the pulmonary artery supplying the systemic circulation (25). In the long-term, increased stress of the pulmonary valve below the systemic pressure may pose a problem. Insufficiency and stenosis may develop. Echocardiography is important to regularly check the potential complications, including, but not limited to the function of these valves, the status of the coronary anastomoses.

\section{Coarctation of the aorta (CoA)}

$\mathrm{CoA}$ is a relatively common congenital heart disease characterized by the pressure overload of the left ventricle. There are different morphologies, the most common form is the focal stenosis of the aorta in the level of the isthmus. Pressure is elevated proximally to the stenosis, while it is decreased distally within the aorta. Rarely, the disease affects the abdominal aorta. It is often accompanied by bicuspid aortic valve, VSD, patent ductus arteriosus and aneurysm of the basal cerebral arteries, arteries of the circle of Willis. The severity of the disease is varying, the patient may have no complaints or symptoms, but the disease may present as a very severe interruption of the aortic arch. Different types of operation are performed: end to end anastomosis, patch plasty repair or graft implantation; subclavian flap aortoplasty; jump grafts, bypassing the coarctation. In other cases, balloon dilatation and stent implantation is performed (26). During the follow-up, evaluation of complaints and symptoms, as well as physical examination and regular blood pressure measurement (on the left and right arm), blood pressure response on exertion and echocardiography are essential. Besides recurrent or residual arterial hypertension, most common long-term complications include recurrence of the stenosis and aneurysm at the surgical site. Sensitivity of transthoracic echocardiography in detecting these lesions is approximately $76 \%$, sensitivity may be increased to even $90 \%$ using colour Doppler and continuous wave Doppler methods (27). Recurrent stenosis may be visualized with $2 \mathrm{D}$ echocardiography using colour Doppler imaging from suprasternal view as turbulent flow (Figure 12). The diameter of the ascending aorta, the supraaortic segments, and the consequent left ventricular hypertrophy can be measured with $2 \mathrm{D}$ echocardiography. In case of restenosis, continuous wave Doppler method may be used to measure the gradient from the velocity, although it should be noted that the continuity equation was validated in case of focal valvular stenoses (28). Recurrent coarctation may be longer, tubular, and spatial rotation may be present, therefore the severity of the stenosis cannot be determined safely with echocardiography in all cases. From suprasternal view, the diastolic "run-off" phenomenon (gradient that can be detected during diastole as well) suggesting severe restenosis can be detected with continuous wave Doppler echocardiography. Real time 3D echocardiography may help detecting the length and spatial location of the stenosis. During the follow-up, another imaging modality 
is recommended for at least once in every patient who had surgery or intervention due to coarctation: MR angiography or CT angiography. Transthoracic echocardiography is suitable to detect the consequent left ventricular hypertrophy (2).

\section{Summary}

Echocardiography is a very useful, easily accessible and reproducible non-invasive imaging method in the follow-up of adult patients with congenital heart diseases. The method is able to detect residual heart disorders and to quantify these lesions. Knowing the benefits and limitations of each method, the special echocardiography method that provides adequate answers to the clinical question during the followup can be selected. It is important to know the limitations of each method as well, so the clinician knows when MRI, CT or invasive method is required to resolve a certain problem or to optimize therapy. Clinical symptoms and the clinical status of the patient have to be considered when deciding whether another intervention or surgery is indicated and in selecting the intervention or surgical technique to be performed. During the follow-up, it is important to evaluate the patient's complaints, clinical status and other diseases, as well as the results of adequately selected and interpreted imaging studies.

\section{Acknowledgements}

None.

\section{Footnote}

Conflicts of Interest: The authors have no conflicts of interest to declare.

\section{References}

1. Ávila P, Mercier LA, Dore A, et al. Adult congenital heart disease: a growing epidemic. Can J Cardiol 2014;30:S410-9.

2. Baumgartner H, Bonhoeffer P, De Groot NM, et al. ESC Guidelines for the management of grown-up congenital heart disease (new version 2010). Eur Heart J 2010;31:2915-57.

3. Marelli AJ, Ionescu-Ittu R, Mackie AS, et al. Lifetime prevalence of congenital heart disease in the general population from 2000 to 2010. Circulation 2014;130:749-56.

4. Steeds RP. Echocardiography: frontier imaging in cardiology. Br J Radiol 2011;84:S237-45.

5. Takeda H, Muro T, Saito T, et al. Diagnostic accuracy of transthoracic and transesophageal echocardiography for the diagnosis of bicuspid aortic valve: comparison with operative findings. Osaka City Med J 2013;59:69-78.

6. Reynolds HR, Jagen MA, Tunick PA, et al. Sensitivity of transthoracic versus transesophageal echocardiography for the detection of native valve vegetations in the modern era. J Am Soc Echocardiogr 2003;16:67-70.

7. Charakida M, Shakeel Q, John MS. 3D Echocardiography for Planning and Guidance of Interventional Closure of VSD. JACC: Cardiovascular Imaging 2013;6:120-3.

8. Muraru D, Badano LP, Vannan M, et al. Assessment of aortic valve complex by three-dimensional echocardiography: a framework for its effective application in clinical practice. Eur Heart J Cardiovasc Imaging 2012;13:541-55.

9. Rong LQ. An update on intraoperative three-dimensional transesophageal echocardiography. J Thorac Dis 2017;9:S271-82.

10. Nemes A, Havasi K, Domsik P, et al. Evaluation of right atrial dysfunction in patients with corrected tetralogy of Fallot using 3D speckle-tracking echocardiography. Insights from the CSONGRAD Registry and MAGYARPath Study. Herz 2015;40:980-8.

11. Havasi K, Kalapos A, Berek K, et al. More than 50 years' experience in the treatment of patients with congenital heart disease at a Hungarian university hospital. Orv Hetil 2015;156:794-800.

12. Forsey J, Friedberg MK, Mertens L. Speckle tracking echocardiography in pediatric and congenital heart disease. Echocardiography 2013;30:447-59.

13. Barker PC. Intracardiac echocardiography in congenital heart disease. J Cardiovasc Transl Res 2009;2:19-23.

14. Budts W, Gewillig M, Van de Werf F. Left-to-right shunting in common congenital heart defects: which patients are eligible for percutaneous interventions? Acta Cardiol 2003;58:199-205.

15. Rao PS, Harris AD. Recent advances in managing septal defects: atrial septal defects. F1000Res 2017;6:2042.

16. Martin SS, Shapiro EP, Mukherjee M. Atrial Septal Defects - Clinical Manifestations, Echo Assessment, and Intervention. Clin Med Insights Cardiol 2015;8:93-8.

17. Cavalcante JL, Lalude OO, Schoenhagen P, et al. Cardiovascular Magnetic Resonance Imaging for Structural and Valvular Heart Disease Interventions. JACC Cardiovasc Interv 2016;9:399-425.

18. Gatzoulis MA, Webb GD, Daubeney PE. Diagnosis and 
management of adult congenital heart disease. 2nd ed. Edinbourgh: Elsevier Saunders, 2011.

19. Mercer-Rosa L, Yang W, Kutty S, et al. Quantifying Pulmonary Regurgitation and Right Ventricular Function in Surgically Repaired Tetralogy of Fallot: A Comparative Analysis of Echocardiography and Magnetic Resonance Imaging. Circ Cardiovasc Imaging 2012;5:637-43.

20. Engelfriet P, Boersma E, Oechslin E, et al. The spectrum of adult congenital heart disease in Europe: morbidity and mortality in a 5 year follow-up period. The Euro Heart Survey on adult congenital heart disease. Eur Heart J 2005;26:2325-33.

21. Warnes CA. Transposition of the great arteries. Circulation 2006;114:2699-709.

22. Konstantinov IE, Alexi-Meskishvili VV, Williams WG, et al. Atrial switch operation: past, present, and future. Ann Thorac Surg 2004;77:2250-8.

23. Cinteza E, Carminati M. Balloon atrial septostomy - almost half a century after. Maedica (Buchar) 2013;8:280-4.

Cite this article as: Havasi K, Ambrus N, Kalapos A, Forster T, Nemes A. The role of echocardiography in the management of adult patients with congenital heart disease following operative treatment. Cardiovasc Diagn Ther 2018;8(6):771-779. doi: 10.21037/cdt.2018.09.11
24. Squarcia U, Macchi C. Transposition of the great arteries. Curr Opin Pediatr 2011;23:518-22.

25. De Praetere H, Vandesande J, Rega F, et al. 20 years of arterial switch operation for simple TGA. Acta Chir Belg 2014;114:92-8.

26. Duara R, Theodore S, Sarma PS, et al. Correction of coarctation of aorta in adult patients--impact of corrective procedure on long-term recoarctation and systolic hypertension. Thorac Cardiovasc Surg 2008;56:83-6.

27. Huang F, Chen Q, Huang WH, et al. Diagnosis of congenital coarctation of the aorta and accompany malformations in infants by multi-detector computed tomography angiography and transthoracic echocardiography: A Chinese clinical study. Med. Sci. Monit 2017;23:2308-14.

28. Giardini A, Tacy TA. Pressure recovery explains Doppler overestimation of invasive pressure gradient across segmental vascular stenosis. Echocardiography 2010;27:21-31. 